OPEN ACCESS

Edited by:

Alan Jay Katz,

St. Francis Hospital, United States

Reviewed by:

Ruijie Yang,

Peking University Third Hospital, China

Jung Sun Yoo,

Hong Kong Polytechnic University,

Hong Kong

*Correspondence:

Shao-yan Liu

shaoyan_liu@sina.com

Jun-lin Yi

yijunlin1969@163.com

Specialty section:

This article was submitted to

Radiation Oncology,

a section of the journal

Frontiers in Oncology

Received: 30 August 2020 Accepted: 23 November 2020

Published: 08 January 2021

Citation:

He Y-q, Zhang X-w, Zhu Y-m, Ni X-g, Huang Z-h, An C-m, Yi J-l and Liu S-y (2021) Posttreatment Non-Improved Vocal Cord Mobility Indicates the Need of Salvage Surgery for Hypopharyngeal Carcinomas.

Front. Oncol. 10:600599. doi: 10.3389/fonc.2020.600599

\section{Posttreatment Non-Improved Vocal Cord Mobility Indicates the Need of Salvage Surgery for Hypopharyngeal Carcinomas}

\author{
Yu-qin $\mathrm{He}^{1}$, Xi-wei Zhang ${ }^{1}$, Yi-ming $\mathrm{Zhu}^{1}{ }^{1}$, Xiao-guang $\mathrm{Ni}^{2}$, Ze-hao Huang ${ }^{1}$, \\ Chang-ming $\mathrm{An}^{1}$, Jun-lin $\mathrm{Yi}^{3{ }^{*}}$ and Shao-yan Liu ${ }^{1 *}$ \\ 1 Department of Head and Neck Surgery, National Cancer Center/National Clinical Research Center for Cancer/Cancer \\ Hospital, Chinese Academy of Medical Sciences, Peking Union Medical College, Beijing, China, ${ }^{2}$ Department of Endoscopy, \\ National Cancer Center/National Clinical Research Center for Cancer/Cancer Hospital, Chinese Academy of Medical \\ Sciences, Peking Union Medical College, Beijing, China, ${ }^{3}$ Department of Radiation Oncology, National Cancer Center/ \\ National Clinical Research Center for Cancer/Cancer Hospital, Chinese Academy of Medical Sciences, Peking Union Medical \\ College, Beijing, China
}

Introduction: We aimed to analyze the relationship between the changed status of vocal cord mobility and survival outcomes.

Methods: Seventy-eight patients with dysfunctional vocal cords and hypopharyngeal carcinomas accepted non-surgical treatment as the initial therapy between May 2009 and December 2016. Vocal cord mobility was assessed before and after the initial non-surgical treatment. The cord mobility status was classified as normal, impaired, and fixed. Patients with improved mobility (IM) ( $n=56$ ) were retrospectively analyzed for disease-free survival (DFS), recurrence-free survival (RFS), and overall survival (OS) and compared with 22 patients with non-improved mobility (non-IM).

Results: Fifty-six (71.8\%) patients had improved cord mobility after the initial non-surgical treatment. The non-improved cord mobility was significantly associated with shortened DFS $(P=0.005)$, RFS $(P=0.002)$, and OS $(P<0.001)$. If non-improved cord mobility was regarded as an indicator for local-regional recurrence within 1 year, the sensitivity and the specificity were $60.9 \%, 87.5 \%$ respectively. The multivariate analysis showed that improved cord mobility $(P=0.006)$ and salvage surgery $(P=0.015)$ were both independent protective factors for OS.

Conclusion: Changes in cord mobility are a key marker for predicting prognosis. Nonimproved cord mobility may indicate a high possibility of a residual tumor, therefore, patients whose cord mobility remains dysfunctional or worsens after non-surgical treatment might need an aggressive salvage strategy.

Keywords: salvage therapy, radiotherapy, hypopharyngeal neoplasms, prognosis, vocal fold paralysis 


\section{INTRODUCTION}

Although the prognosis of hypopharyngeal squamous cell carcinoma (HPSCC) has improved in the last decade, HPSCC is still associated with the worst survival outcomes among all head and neck cancers. The 5-year survival rate of patients with advanced disease (stages III-IV) ranges from 30 to $54 \%(1,2)$. Pretreatment vocal cord fixation is a significantly poor prognostic factor in HPSCC. According to the $8^{\text {th }}$ American Joint Committee Cancer (AJCC) staging atlas (3), hemilarynx fixation is one of the indicators used to upgrade T1-2 to T3-4 regardless of the size of the primary tumor. The mobility of the vocal cord might affect the choice of conservative surgery.

Although advanced HPSCC has no standard treatment strategy, it is no doubt that advanced HPSCC should be treated with combined therapy by a multidisciplinary team. Traditional open surgery is always followed by unacceptable functional loss. Therefore, the treatment strategy is gradually shifting from surgery-based treatment to radiotherapy-based treatment for organ preservation. However, salvage surgery still plays an important role in the treatment of advanced HPSCC and relapse, but the proper time for surgical invention and how to select candidates who would benefit from the sacrifice of function for survival purposes remain questionable.

In clinical practice, we have observed that the cord mobility status might change after non-surgical treatment in patients with laryngeal and hypopharyngeal cancers. A few studies have analyzed this phenomenon in patients with laryngeal cancers, but the authors did not agree on whether remobility of the vocal cord could predict a good prognosis $(3,4)$. No literature has focused on the value of improved cord mobility after non-surgical therapy in HPSCC. It is unclear whether improved cord mobility could be a prognostic signal or a predictive factor of therapeutic efficacy. In other words, we wonder whether non-improved cord mobility indicates a residual tumor after non-surgical therapy and the need for salvage surgery. To the best of our knowledge, this is the first study to evaluate the prognostic value of a changed vocal cord mobility status after non-surgical therapy in HPSCC. We also evaluated the role of salvage surgery in survival outcomes.

\section{MATERIALS AND METHODS}

\section{Patients}

This retrospective study focused on patients with dysfunctional vocal cords and HPSCC. All patients accepted non-surgical treatment as the initial therapy at the National Cancer Center (China) between May 2009 and December 2016. The study was approved by the institutional review board of the National Cancer Hospital of the Chinese Academy of Medical Sciences, Beijing, China. All patients wrote informed consent before treatment. Each therapeutic strategy was discussed by a multidisciplinary team before treatment. Non-surgical treatment included a radiotherapybased strategy with or without systemic therapy. Systemic therapy included concurrent or neoadjuvant. Patients who could not tolerate a total dose of 66-70 Gy and who had distant metastasis at the initial diagnosis were excluded. There were no toxicity-mandated breaks or delays during radiotherapy. Salvage surgery included at least one of the following: neck dissection, partial pharyngectomy, and total pharyngolaryngectomy. Tumor (T) classification and lymph node (N) classification were defined through imaging examinations and fiberoptic laryngoscopy according to the criteria of the AJCC $7^{\text {th }}$ edition staging system (5).

\section{Vocal Cord Mobility}

We reviewed the results of fiberoptic laryngoscopy in all enrolled patients. All classifications of the status of vocal cord mobility were carried out by one experienced endoscopist (who performed fiberoptic laryngoscopy over 3 years). Vocal cord mobility was assessed at least twice, including before and within 3 months after the initial non-surgical treatment. The cord mobility status was classified as normal or dysfunctional mobility. Dysfunctional cord mobility included impaired and fixed cord mobility. The impaired cord mobility was defined as the mobility of vocal cord weaken than that of the healthy side. Improved mobility (IM) consisted of complete response (CR) and partial response (PR). CR was defined as cord mobility that changed from impaired/fixed to normal after non-surgical treatment, and PR was defined as cord mobility that changed from fixed to impaired mobile after nonsurgical treatment. If the mobility status of the patient remained fixed/impaired after non-surgical treatment, it was defined as stable dysfunction (SD). If the mobility status of the patient changed from impaired to fixed after non-surgical treatment, it was defined as progressive dysfunction (PD). PD and SD were both regarded as non-improved mobility (non-IM).

\section{Survival Outcomes}

Outcomes consisted of recurrence-free survival (RFS), diseasefree survival (DFS), and overall survival (OS). OS was calculated from the date of the completion of initial non-surgical treatment and was censored at the date of all-cause death or the last followup. Local-regional recurrence, new distant metastasis, and persistent disease were censored as recurrence. New secondary primary cancers and recurrence were end events of DFS and were diagnosed by an imaging examination or biopsy. If patients did not accept salvage surgery, RFS and DFS were calculated from the date of the completion of initial non-surgical treatment; If patients accepted salvage surgery, RFS and DFS were calculated from the date of salvage surgery. Imaging examinations have done on all individuals every 3 months for the first 2 years, followed by every 6 months for the next 3 years, and annually thereafter. Based on the results, salvage surgeries have performed to the patients who were confirmed stable disease, incomplete response or relapse after radiotherapy. All salvage surgery should be performed within 6 months after non-surgical treatment. Salvage surgery beyond 6 months and with positive pathological results was regarded as local-regional recurrence.

\section{Statistical Analysis}

All categorical variables were estimated using two-sided Fisher's exact tests, and all continuous variables were estimated using the Wilcoxon rank-sum test. Overall, recurrence-free and diseasefree survival curves were obtained utilizing Kaplan-Meier. 
Associations between prognostic factors and survival outcomes were tested on univariate and multivariate Cox models. In multivariate analysis, cox-proportional hazard regression analyses were performed in variables with $P$ values $<0.15$ on univariate analyses. $P$-value less than 0.05 indicates statistical significance. All statistical analyses were performed on SPSS 26.0 software (IBM, Armonk, NY).

\section{RESULTS}

\section{Risk Factors Associated With the Recovery of Cord Mobility}

A total of 78 patients with HPSCC were included in this study. There were 75 males and 3 females, with a mean age of 57.5 years (range 36-80 years). Before the initial treatment, $56(71.8 \%)$ patients had fixed vocal cords, and $22(28.2 \%)$ patients had impaired vocal cords. Fifty-six (71.8\%) patients had improved cord mobility after non-surgical treatment. The rates of SD, PD, PR, and CR were 24.4\% (19/78), 3.8\% (3/78), 12.8\% (10/78), and $59.0 \%(46 / 78)$, respectively. Details of demographic characteristics between the IM and non-IM groups are summarized in Table 1. Patients who had no clinical evidence of lymph node metastasis ( $\mathrm{cN} 0)(P=0.016)$ and who did not undergo pretreatment tracheotomy $(P<0.001)$ were more likely to have IM after non-surgical treatment than patients with clinical evidence of lymph node metastasis and those who underwent pretreatment tracheotomy. Patients with postcricoid region cancers had a tendency of having $I M$, but the difference did not reach statistical significance $(P=0.080)$.

TABLE 1 | Demographic characteristics between the improved mobility (IM) and non-IM groups.

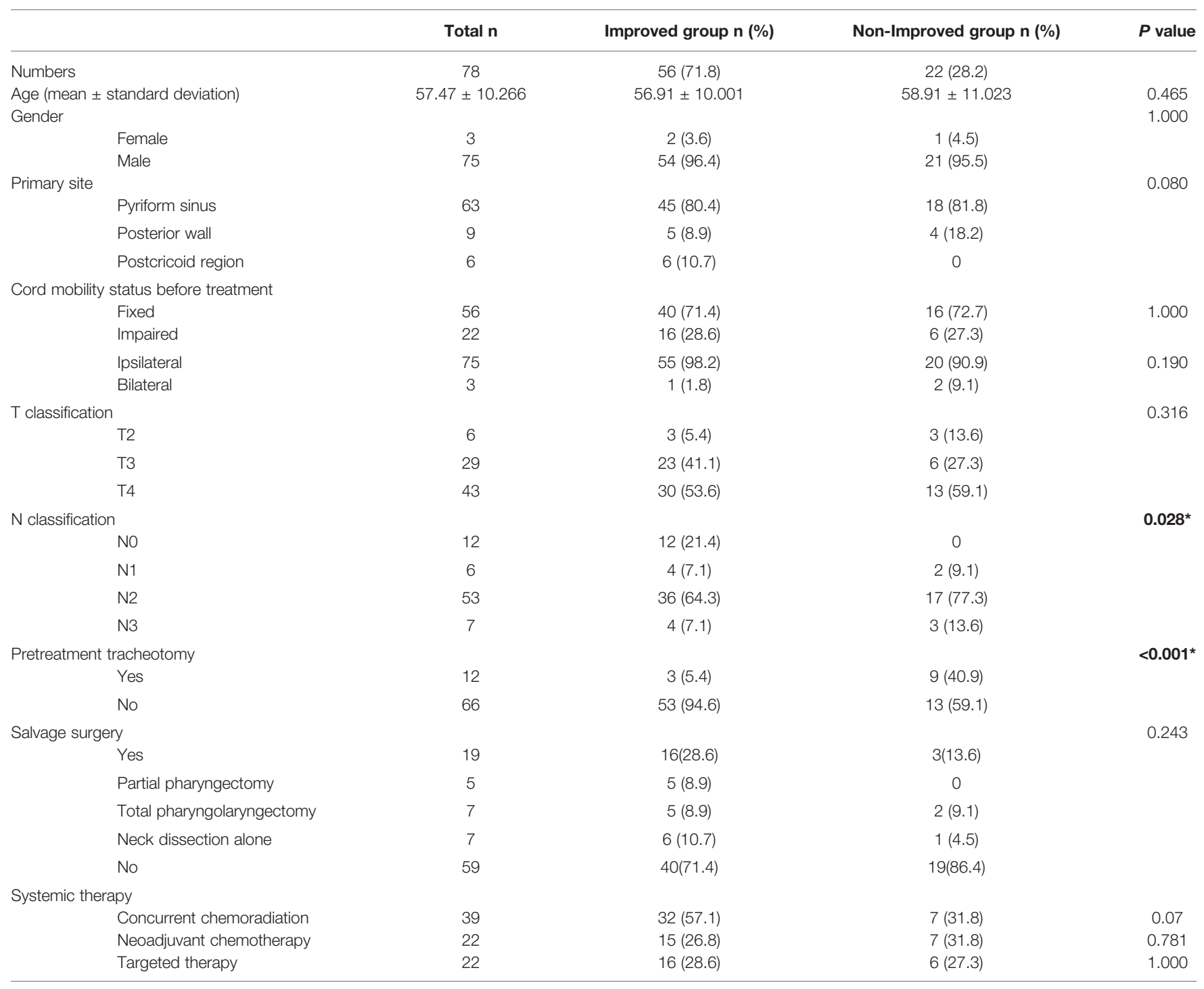

${ }^{*} P<0.05$.

All $p$ value below 0.05 were highlighted in bold values. 


\section{Outcomes Between Two Groups}

Six patients were lost to follow-up after non-surgical treatment: two in the non-IM group and four in the IM group. Eight patients occurred heterochronic secondary primary cancers after initial treatment, seven patients had esophageal carcinomas and one patient had soft palate carcinoma. Twenty-three patients $(44.2 \%)$ had recurrence in IM group comparing to 16 patients $(76.2 \%)$ in non-IM group (Table 2). Non-improved cord mobility was significantly associated with recurrence $(P=0.019)$, especially local-regional recurrence $(P=0.001)$. In patients who did not accept salvage surgery, if non-improved cord mobility was regarded as an indicator for local-regional recurrence within 1 year, the sensitivity, the specificity, positive predictive value, and negative predictive value were $60.9,87.5$, $77.8,75.7 \%$ respectively.

With a median follow-up of 33.5 months (range 1-130 months), the total 5-year OS rates were $52.4 \%$, respectively. The non-improved cord mobility was significantly associated with shortened DFS $(P=0.005)$, RFS $(P=0.002)$, and OS $(P<0.001)$ (Figures 1-3). The 1year DFS rate of the non-IM group was $27.2 \%$. The 5-year RFS and OS rates of the non-IM group (compared to the IM group) were $21.8 \%$ (vs. $53.2 \%$ ) and $30.0 \%$ (vs. $61.5 \%$ ), respectively.

TABLE 2 | Outcomes between improved mobility (IM) and non-IM groups.

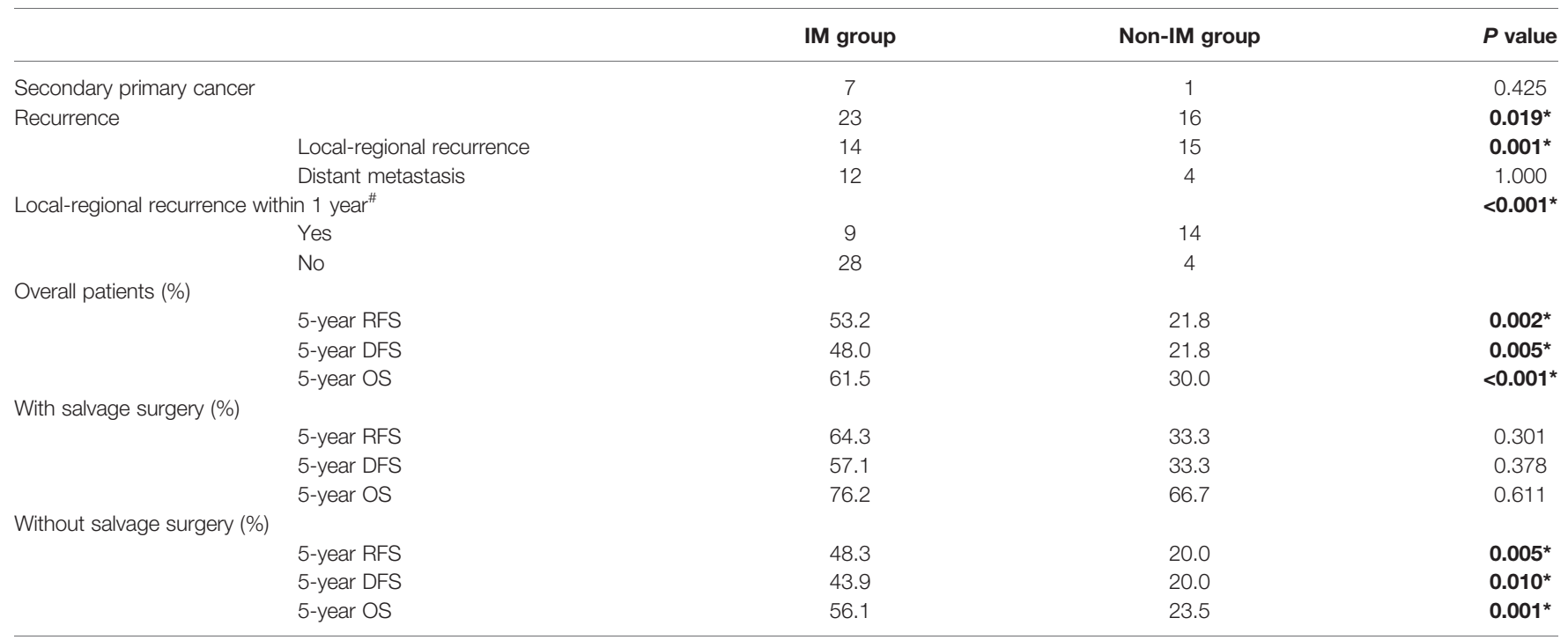

${ }^{\#}$ Patients, who did not accept salvage surgery, occurred local-regional recurrence or not. ${ }^{*} P<0.05$; All $p$ value below 0.05 were highlighted in bold values.

OS, overall survival; RFS, recurrence-free survival; DFS, disease-free survival; IM, improved cord mobility; non-IM, non-improved cord mobility.

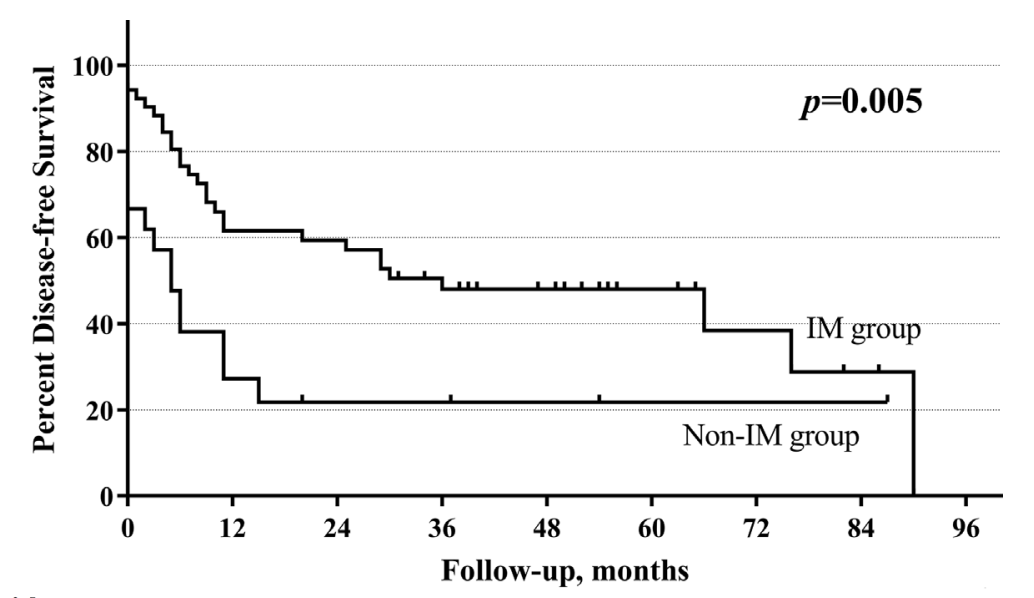

\begin{tabular}{|c|c|c|c|c|c|c|c|c|c|}
\hline \multirow{2}{*}{\multicolumn{10}{|c|}{ No. at risk }} \\
\hline & & & & & & & & & \\
\hline IM & 52 & 30 & 28 & 20 & 15 & 9 & 5 & 3 & 0 \\
\hline Non-IM & 21 & 7 & 4 & 4 & 3 & 2 & 2 & 2 & 0 \\
\hline
\end{tabular}

FIGURE 1 | Disease-free survival curve of improved mobility (IM) group and non-IM group. 


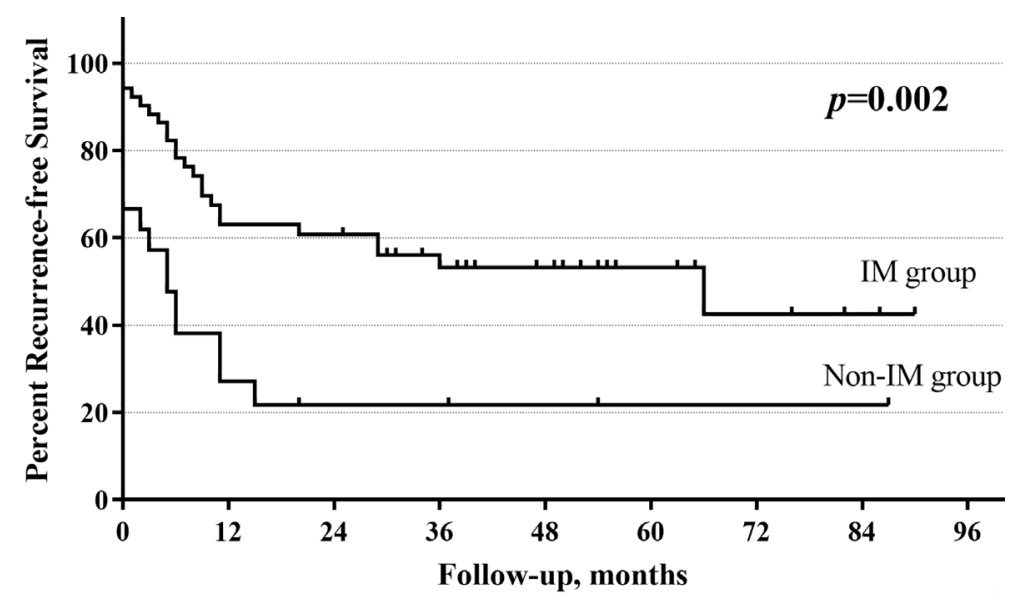

$\begin{array}{lrrrrrrrrr}\text { No. at risk } & & & & & & & & & \\ \text { IM } & 52 & 30 & 28 & 20 & 15 & 9 & 5 & 0 \\ \text { Non-IM } & 21 & 7 & 4 & 4 & 3 & 2 & 2 & 2 & 0\end{array}$

FIGURE 2 | Recurrence-free survival curve of improved mobility (IM) group and non-IM group.

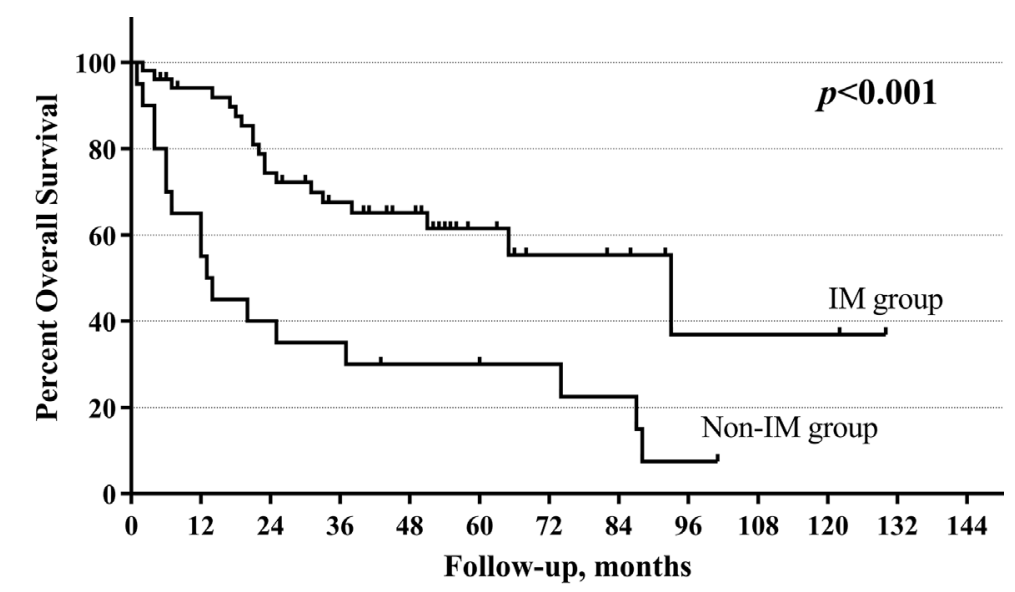

$\begin{array}{lrrrrrrrrrrrr}\text { No. at risk } & & & & & & & & & & & & \\ \text { IM } & 52 & 45 & 36 & 29 & 23 & 12 & 7 & 6 & 3 & 0 & & \\ \text { Non-IM } & 20 & 13 & 9 & 8 & 6 & 5 & 5 & 4 & 2 & 1 & 1 & 0\end{array}$

FIGURE 3 | Overall survival curve of improved mobility (IM) group and non-IM group.

\section{Multivariate Analysis of Recurrence-Free Survival, Disease-Free Survival, and Overall Survival}

The univariate analysis revealed that a high $\mathrm{N}$ classification $(P=0.016)$, without salvage surgery $(P=0.010)$, non-improved cord mobility $(P<0.001)$, pretreatment tracheotomy $(P=0.046)$ were associated with poor OS. Furthermore, the multivariate analysis revealed that all of these variables except for pretreatment tracheotomy were independent risk factors (Table 3). The mean OS time was 78 months in the IM group vs. 34 months in the non-IM group (HR 2.734, 95\% $\mathrm{CI}=1.340-5.578$ ). The multivariate analysis also revealed that IM was an independent protective factor for prolonged DFS $(P=0.043)$ and RFS $(P=0.030)$. The median DFS time was 36 months in the IM group vs. 5 months in the non-IM group (HR 1.925, 95\% CI=1.020-3.636).

\section{Salvage Surgery as a Protective Factor for Overall Survival}

The multivariate analysis revealed that patients who underwent salvage surgery had higher OS (HR 0.262, 95\% CI $=0.089-0.772$, $P=0.015)$ than patients who did not undergo salvage surgery. IM group had obviously higher 5-year RFS, DFS, and OS not only in overall cohort but also in cohort without salvage surgery (Table 2). However, in cohort with salvage surgery, there were 
TABLE 3 | Univariate and multivariate analysis about disease-free survival (DFS), recurrence-free survival (RFS), and overall survival (OS).

\begin{tabular}{|c|c|c|c|c|c|c|c|c|c|}
\hline & \multicolumn{3}{|c|}{ Univariate analysis } & \multicolumn{6}{|c|}{ Multivariate analysis } \\
\hline & \multirow{2}{*}{$\frac{\text { DFS }}{P \text { value }}$} & \multirow{2}{*}{$\begin{array}{c}\text { RFS } \\
P \text { value }\end{array}$} & \multirow{2}{*}{$\frac{\text { OS }}{P \text { value }}$} & \multicolumn{2}{|l|}{ DFS } & \multicolumn{2}{|l|}{ RFS } & \multicolumn{2}{|l|}{ OS } \\
\hline & & & & $\mathrm{HR}(95 \% \mathrm{Cl})$ & $P$ value & HR (95\% Cl) & $P$ value & HR (95\% Cl) & $P$ value \\
\hline $\mathrm{N}$ classification & $<0.001^{*}$ & $<0.001^{\star}$ & $0.016^{\star}$ & $1.803(1.033-3.145)$ & $0.038^{\star}$ & $2.016(1.066-3.813)$ & $0.031^{*}$ & $2.611(1.354-5.036)$ & $0.004^{*}$ \\
\hline Pretreatment tracheotomy & 0.362 & 0.285 & $0.046^{\star}$ & / & / & / & / & / & 0.228 \\
\hline Primary site & 0.820 & 0.629 & 0.712 & / & / & / & / & / & / \\
\hline $\mathrm{IM} /$ non-IM & $0.005^{\star}$ & $0.002^{*}$ & $<0.001^{*}$ & 1.925 (1.020-3.636) & $0.043^{\star}$ & $2.074(1.075-4.002)$ & $0.030^{*}$ & $2.734(1.340-5.578)$ & $0.006^{*}$ \\
\hline Salvage surgery & 0.225 & 0.138 & $0.010^{\star}$ & / & / & / & 0.262 & $0.262(0.089-0.772)$ & $0.015^{\star}$ \\
\hline Concurrent chemoradiation & 0.352 & 0.303 & 0.677 & / & / & / & / & / & / \\
\hline
\end{tabular}

OS, overall survival; RFS, recurrence-free survival; DFS, disease-free survival; IM, improved cord mobility; non-IM, non-improved cord mobility.

$\mathrm{HR}$, hazard ratio; $\mathrm{Cl}$, confidence interval; ${ }^{*} \mathrm{P}<0.05$. All $\mathrm{p}$ value below 0.05 were highlighted in bold values.

no significant difference in all kinds of survival between two groups.

Patients were divided into four groups according to the change in cord mobility and the choice of salvage surgery. The mean OS times of these four groups were as follows (from high to low): IM with salvage surgery (105 months), non-IM with salvage surgery (71 months), IM without salvage surgery (67 months), and non-IM without salvage surgery (28 months) $(\mathrm{P}<0.001)$. Figure 4 showed the overall survival curve in four groups.

\section{DISCUSSION}

Although HPSCC is rare (incidence of 5\%), it has the worst prognosis of all head and neck squamous cell carcinomas $(1,2,6)$.
Pretreatment cord dysfunction is regarded as an indicator of an unfavorable prognosis $(7,8)$. However, the cord mobility status might change after non-surgical treatment. Vocal cord dysfunction might be due to tumor invasion of laryngeal structures, such as the cricoarytenoid joint, thyroarytenoid muscle, and posterior cricoarytenoid muscles, and tumor invasion of recurrent nerves. However, the most common cause of vocal cord immobility can be explained by the weight effect (i.e., the occupancy of the tumor mass). Katilmis et al. (9) found that $50 \%$ of vocal cord dysfunction cases were caused by the weight effect because none of the laryngeal structures were involved in these cases based on the histopathological evaluation of total laryngectomy specimens. Therefore, when the tumor mass was eliminated through non-surgical treatment, patients who experienced vocal cord dysfunction caused by the weight effect had a high possibility of experiencing normal mobility. We
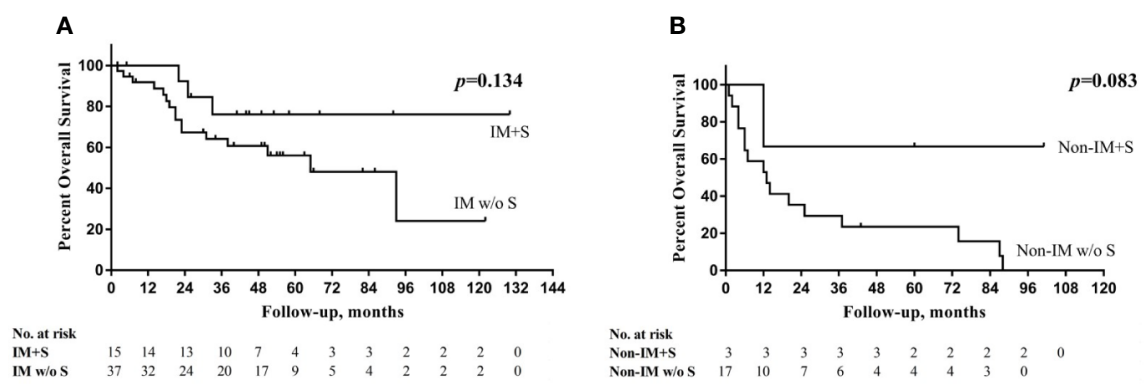

C

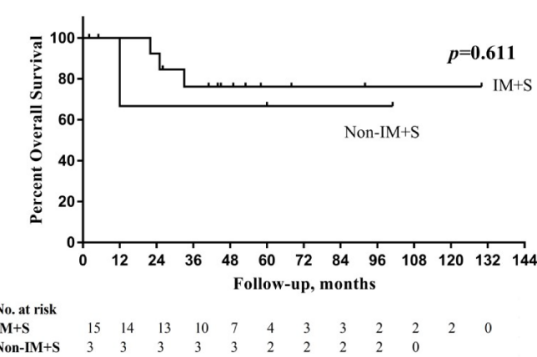

D

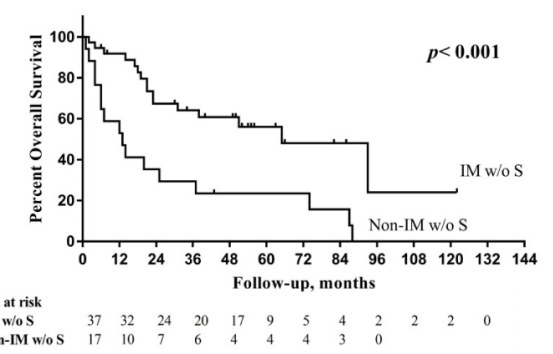

FIGURE 4 | (A) Overall survival (OS) curve in IM group with or without salvage surgery (S); (B) OS curve in non-IM group with or without S; (C) OS curve in IM group with $S$ and non-IM group with S; (D) OS curve in IM group without S and non-IM group without S. 
speculate that the weight effect might partly explain why the rate of improved cord mobility was as high as $71.8 \%$ after nonsurgical treatment in our study.

Although few studies have evaluated the relationship between changes in the cord mobility status and prognosis in HPSCC, some studies that focused on patients with laryngeal carcinoma and improved cord mobility might provide some insights. Solares et al. (4) analyzed the 5-year local control rate of 23 patients with advanced laryngeal carcinoma who had initial vocal cord fixation and then accepted concurrent chemoradiotherapy. Fifteen patients with improved cord mobility had a 100\% 5-year OS rate, whereas those with persistent fixed cord mobility after treatment had a $25 \% 5$-year OS rate $(P<0.001)$. However, not all evidence suggests that IM is a predictive factor of good local control. Lee et al. (10) performed a retrospective study on 69 patients with dysfunctional vocal cords and laryngeal cancers. They did not find a significant difference in the 2-year local control rate between patients with IM (53/69) and those without IM (16/69) (70\% vs. 77\%, $P=0.81)$. Different from the studies mentioned above, our target population was patients with HPSCC. According to our results, non-improved cord mobility was a strong risk factor for RFS, DFS, and OS. The improved mobility group had a mean OS time of 78 months, which was twice as long as that of the non-IM group. Furthermore, the IM group had a median DFS time seven times longer than that of the non-IM group (36 months vs. 5 months). In contrast, nonimproved cord mobility might be an indicator for a high possibility of a residual tumor. If non-improved cord mobility was used to predict local-regional recurrence after non-surgical treatment within 1 year, it had a high specificity $(87.5 \%)$, although the sensitivity was $60.9 \%$. Therefore, we hypothesized that non-improved cord mobility might be a key indicator that should be evaluated to assess the response to non-surgical treatment in addition to imaging examinations, such as contrasted computed tomography (CT), magnetic resonance imaging (MRI), and fluorodeoxyglucose positron emission tomography (FDG-PET). Patients whose cord mobility remained dysfunctional or worsened after non-surgical treatment might need a more aggressive salvage strategy for survival benefits and local control.

The trend in the management of HPSCC is toward a preference for organ preservation without sacrificing survival. However, salvage surgery still has an obvious advantage in the survival of advanced HPSCC patients, especially in those who experience recurrence (11). Al-Mamgani et al. (12) revealed that the total laryngectomy-based strategy resulted in better 5-year local control for T4 laryngeal and hypopharyngeal cancers than organ-preservation chemoradiation (64\% vs. 87\%, $P=0.030)$; however, there was no improvement in OS. In the current study, salvage surgery had an independently positive impact on OS in HPSCC patients with pretreatment dysfunctional cord mobility. In the non-IM group, salvage surgery increased the 5year OS rate from 23.5 to $66.7 \%$. Non-IM group had obviously shorter 5-year RFS, DFS, and OS not only in overall cohort but also in cohort without salvage surgery. However, in cohort with salvage surgery, there were no significant difference between two groups in all kinds of survival outcomes and the 5-year OS of non-IM group became closed to IM group (66.7\% vs. $76.2 \%$, $P=0.611$ ). We speculate that patients with non-IM might obtain a survival benefit from salvage surgery, but we cannot draw a conclusion because of the small sample size.

When predicting the prognosis of HPSCC, several risk factors, such as $\mathrm{T}$ classification, $\mathrm{N}$ classification, pretreatment cord fixation, and pretreatment tracheotomy dependence, should be considered (13). Ho et al. (14) retrospectively analyzed the survival rate of 8,351 patients with hypopharyngeal and laryngeal cancers. Their univariate and multivariable models suggested that metastatic lymph node burden and extranodal extension were two crucial risk factors for mortality. In accordance with the previous study, our results demonstrated that $\mathrm{N}$ classification was an independent risk factor for OS, RFS, and DFS. In a multiinstitutional study containing 226 patients with advanced laryngeal cancers, $31.4 \%(71 / 226)$ underwent pretreatment tracheotomy. Moreover, their results showed that patients who underwent pretreatment tracheotomy had a statistically significant decrease in both OS (HR 1.55, 95\% CI=1.03-2.34, $P=0.03$ ) and DFS (HR 1.54, 95\% CI $=2.07-2.22, P=0.02)(15)$. Some studies have also revealed that pretreatment tracheotomy was a strong predictor for long-term tracheostomy after organpreservation strategy in laryngeal and hypopharyngeal cancers $(16,17)$. In our study, which focused on HPSCC patients with pretreatment cord dysfunction, pretreatment tracheotomy was associated with OS only in the univariate analysis, but the difference was not statistically significant in the multivariate analysis. Interestingly, we found that $\mathrm{N}$ classification and pretreatment tracheotomy were two pretreatment predictors associated with the possibility of improved cord mobility. Patients with N0 classification and who did not undergo pretreatment tracheotomy were likely to experience improved cord mobility after non-surgical treatment.

This study had certain limitations. First, since our target population was HPSCC patients with pretreatment dysfunctional cord mobility, the results cannot be extensively applied to all patients with HPSCC. Additionally, there were few females in the study. Hypopharyngeal cancers are more common in the male gender. The prevalence of hypopharyngeal cancer in male gender was shown much higher based on our findings, consistent with that in other literatures from Asian (18-20). Our conclusion may be more applicable for the male patients due to the unbalanced gender distribution. Second, due to the small sample size, we could not perform further analyses to ensure that salvage surgery had a certain survival benefit for patients without improved cord mobility. Last but not least, limited by the retrospective nature and the long inclusion time, this study could not avoid bias from the preference of patients and doctors. Moreover, because of the retrospective nature of the study, we were unable to evaluate the functional outcomes. Additional evidence from randomized controlled trials is required to evaluate the prognostic value of salvage surgery for HPSCC patients without improved cord mobility. The further study that focused on the relationship between improved cord mobility and functional outcomes should also be considered. 


\section{CONCLUSION}

The change in cord mobility should be evaluated after nonsurgical treatment because non-improved cord mobility is a key indicator to predict poor prognosis. In total, $71.8 \%$ of patients with fixed/impaired vocal cords pretreatment improved after non-surgical treatment. Improved cord mobility and salvage surgery both had an independently positive impact on OS in HPSCC patients with pretreatment dysfunctional cord mobility. Non-improved cord mobility may indicate a high possibility of a residual tumor, therefore, patients whose cord mobility remains dysfunctional or worsens after non-surgical treatment might need an aggressive salvage strategy.

\section{DATA AVAILABILITY STATEMENT}

The raw data supporting the conclusions of this article will be made available by the authors, without undue reservation.

\section{ETHICS STATEMENT}

The studies involving human participants were reviewed and approved by the institutional review board of the National Cancer Hospital of the Chinese Academy of Medical Sciences. The patients/participants provided their written informed consent to participate in this study.

\section{REFERENCES}

1. Suzuki M, Fujii T, Yoshii T, Otozai S, Kitamura K, Kanamura R, et al. Trends in the Detail of the Stage and Survival Rate in Hypopharyngeal Cancer over 20 Years. Nihon Jibiinkoka Gakkai kaiho (2016) 119(7):949-54. doi: 10.3950/ jibiinkoka.119.949

2. Petersen JF, Timmermans AJ, van Dijk BAC, Overbeek LIH, Smit LA, Hilgers FJM, et al. Trends in treatment, incidence and survival of hypopharynx cancer: a 20-year population-based study in the Netherlands. Eur Arch Oto Rhino Laryngol: Off J Eur Fed Oto Rhino Laryngol Societies (EUFOS): Affiliated German Soc Oto Rhino Laryngol - Head Neck Surg (2018) 275(1):181-9. doi: 10.1007/s00405-017-4766-6

3. Amin MB, Gress DM. AJCC Cancer Staging Manual. Eight Edition. New York: Springer (2017).

4. Solares CA, Wood B, Rodriguez CP, Lorenz RR, Scharpf J, Saxton J, et al. Does vocal cord fixation preclude nonsurgical management of laryngeal cancer? Laryngoscope (2009) 119(6):1130-4. doi: 10.1002/lary.20225

5. Edge SB, Byrd D, Compton CC, Fritz AG, Greene FL, Trotti A. AJCC Cancer Staging Manual. Seven Edition. NewYork: Springer (2010).

6. Takes RP, Strojan P, Silver CE, Bradley PJ, Haigentz M Jr, Wolf GT, et al. Current trends in initial management of hypopharyngeal cancer: the declining use of open surgery. Head Neck (2012) 34(2):270-81. doi: 10.1002/hed.21613

7. Eiband JD, Elias EG, Suter CM, Gray WC, Didolkar MS. Prognostic factors in squamous cell carcinoma of the larynx. Am J Surg (1989) 158(4):314-7. doi: 10.1016/0002-9610(89)90123-2

8. Pradier R, González A, Matos E, Loria D, Adan R, Saco P, et al. Prognostic factors in laryngeal carcinoma. Experience in 296 male patients. Cancer (1993) 71(8):2472-6. doi: 10.1002/1097-0142(19930415)71:8<2472::AIDCNCR2820710808>3.0.CO;2-Y

\section{AUTHOR CONTRIBUTIONS}

$\mathrm{Y}-\mathrm{qH}$ : study design, data acquisition, data analysis and interpretation, statistical analysis, manuscript preparation, manuscript editing, manuscript review, approval of final manuscript, agrees to be accountable. X-wZ: data acquisition, data analysis, manuscript editing; manuscript review, approval of final manuscript, agrees to be accountable. Y-mZ: study concept, manuscript editing, manuscript review, approval of final manuscript, agrees to be accountable. X-gN: data acquisition, manuscript editing, manuscript review, approval of final manuscript, agrees to be accountable. Z-hH: data acquisition, manuscript editing, manuscript review, approval of final manuscript, agrees to be accountable. C-mA: data analysis, manuscript editing, manuscript review, approval of final manuscript, agrees to be accountable. J-lY: study concept, data analysis, manuscript editing, manuscript review, approval of final manuscript, agrees to be accountable. S-yL: study concept, study design, manuscript editing, manuscript review, approval of final manuscript, agrees to be accountable. All authors contributed to the article and approved the submitted version.

\section{FUNDING}

This research was supported by the Non-profit Central Research Institute fund of Chinese Academy of Medical Sciences. Grant/ Award Number: Grant No.2019-RC-HL-004.

9. Katilmis H, Ozturkcan S, Ozdemir I, Adadan I, Tunc A, Akder A, et al. A clinico-pathological study of laryngeal and hypopharyngeal carcinoma: correlation of cord-arytenoid mobility with histopathologic involvement. Otolaryngol Head Neck Surg: Off J Am Acad Otolaryngol Head Neck Surg (2007) 136(2):291-5. doi: 10.1016/j.otohns.2006.08.022

10. Lee WT, Yoo DS, Puscas L, Witsell D, Cohen SM, Fisher SR, et al. Treatmentinduced changes in vocal cord mobility and subsequent local recurrence after organ preservation therapy for laryngeal carcinoma. Head Neck (2012) 34 (6):792-6. doi: 10.1002/hed.21813

11. Chu PH, Tsang NM, Lee LA, Liao CT, Fang TJ. To do or not to do: salvage management for hypopharyngeal cancer after chemoradiation therapy. Eur Arch Oto Rhino Laryngol: Off J Eur Fed Oto Rhino Laryngol Societies (EUFOS): Affiliated German Soc Oto Rhino Laryngol - Head Neck Surg (2018) 275 (8):2119-26. doi: 10.1007/s00405-018-5042-0

12. Al-Mamgani A, Navran A, Walraven I, Schreuder WH, Tesselaar MET, Klop WMC. Organ-preservation (chemo)radiotherapy for T4 laryngeal and hypopharyngeal cancer: is the effort worth? Eur Arch Oto Rhino Laryngol: Off J Eur Fed Oto Rhino Laryngol Societies (EUFOS): Affiliated German Soc Oto Rhino Laryngol - Head Neck Surg (2019) 276(2):575-83. doi: 10.1007/s00405018-5241-8

13. Genden EM. Head and Neck Cancer - Management and Reconstruction. 2nd ed. New York: Thieme (2019).

14. Ho AS, Kim S, Tighiouart M, Gudino C, Mita A, Scher KS, et al. Association of Quantitative Metastatic Lymph Node Burden With Survival in Hypopharyngeal and Laryngeal Cancer. JAMA Oncol (2018) 4(7):985-9. doi: 10.1001/jamaoncol.2017.3852

15. Byrd SA, Xu MJ, Cass LM, Wehrmann DJ, Naunheim M, Christopher K, et al. Oncologic and functional outcomes of pretreatment tracheotomy in advanced laryngeal squamous cell carcinoma: A multi-institutional analysis. Oral Oncol (2018) 78:171-6. doi: 10.1016/j.oraloncology.2018.01.018 
16. Jefferson GD, Wenig BL, Spiotto MT. Predictors and outcomes for chronic tracheostomy after chemoradiation for advanced laryngohypopharyngeal cancer. Laryngoscope (2016) 126(2):385-91. doi: 10.1002/lary.25585

17. Heukelom J, Navran A, Gouw ZAR, Tesselaar ME, Zuur CL, van Werkhoven E, et al. Organ Function Preservation Failure after (Chemo)Radiotherapy in Head and Neck Cancer: A Retrospective Cohort Analysis. Otolaryngol Head Neck Surg: Off J Am Acad Otolaryngol Head Neck Surg (2019) 161(2):288-96. doi: 10.1177/0194599819846073

18. Kim SY, Rho YS, Choi EC, Kim MS, Woo JH, Lee DH, et al. Clinicopathological factors influencing the outcomes of surgical treatment in patients with T4a hypopharyngeal cancer. BMC Cancer (2017) 17:904. doi: 10.1186/s12885-017-3880-6

19. Kim JW, Kim MS, Kim SH, Kim JH, Lee CG, Kim GE, et al. Definitive Chemoradiotherapy Versus Surgery Followed by Adjuvant Radiotherapy in Resectable Stage III/IV Hypopharyngeal Cancer. Cancer Res Treat (2016) 48:45-53. doi: 10.4143/crt.2014.340
20. Lo WC, Wu CT, Wang CP, Yang TL, Lou PJ, Ko JY, et al. Lymph Node Ratio Predicts Recurrence and Survival for Patients with Resectable Stage 4 Hypopharyngeal Cancer. Ann Surg Oncol (2017) 24:1707-13. doi: 10.1245/ s10434-017-5770-1

Conflict of Interest: The authors declare that the research was conducted in the absence of any commercial or financial relationships that could be construed as a potential conflict of interest.

Copyright (c) $2021 \mathrm{He}$, Zhang, Zhu, Ni, Huang, An, Yi and Liu. This is an openaccess article distributed under the terms of the Creative Commons Attribution License (CC BY). The use, distribution or reproduction in other forums is permitted, provided the original author(s) and the copyright owner(s) are credited and that the original publication in this journal is cited, in accordance with accepted academic practice. No use, distribution or reproduction is permitted which does not comply with these terms. 